\title{
Führen ohne Hierarchie: Macht, Vertrauen und Verständigung im Konzept des Lateralen Führens
}

\author{
Stefan Kühl
}

\begin{abstract}
Zusammenfassung
In Organisationen gibt es immer mehr Anlässe, in denen hierarchische Weisungsbefugnisse nicht mehr zur Verfügung stehen. Hier greift das Konzept des Lateralen Führens, mit dem Verständigungsprozesse organisiert, Machtarenen gebildet und Vertrauensbeziehungen gefördert werden können. Der Artikel zeigt, wie Verständigung, Macht und Vertrauen in Organisationen ineinandergreifen, welche Wechselbeziehungen mit der Formalstruktur der Organisation bestehen und wie in Veränderungsprozessen von Lateraler Führung profitieren können.
\end{abstract}

Die Selbstverständlichkeit, mit der die Hierarchie in Unternehmen, Verwaltungen und Verbänden akzeptiert wurde, scheint schon seit längerer Zeit dahin zu sein. Relevante Informationen fallen häufig nicht nur an der Spitze, sondern in den verschiedensten Bereichen der Organisation an. Diese Informationen können trotz mehr oder minder effektiver Managementinformationssysteme nicht alle nach oben weitergereicht und dort bearbeitet werden. Deswegen wird darauf gesetzt, dass selbst komplexe Probleme relativ weit unten in der Organisation bearbeitet werden - auch mit der Hoffnung, dass darüber die Qualität der Entscheidungen verbessert werden kann.

Natürlich wäre es naiv, Hierarchie insgesamt als ein auslaufendes Modell zu beschreiben oder gar davon auszugehen, dass Hierarchien abgerissen, auseinandergebaut und zerstückelt werden. Man kann mit guten Gründen davon ausgehen, dass es, solange es Organisationen gibt, auch Hierarchien geben wird. Aber der Eindruck gerade von Managern,

S. Kühl $(\bowtie)$

Metaplan Hamburg, Quickborn, Deutschland

E-Mail: StefanKuehl@metaplan.com

(C) Springer Fachmedien Wiesbaden 2016

O. Geramanis, K. Hermann (Hrsg.), Führen in ungewissen Zeiten,

uniscope. Publikationen der SGO Stiftung, DOI 10.1007/978-3-658-11227-1_16 
dass hierarchische Steuerung in Entscheidungsprozessen nur noch begrenzt wirkt, kann nicht ohne weiteres zurückgewiesen werden (siehe dazu Kühl 2015a).

Das Konzept des Lateralen Führens greift diese Problematik auf und entwickelt Führungsansätze jenseits der hierarchischen Steuerung. Es geht beim Lateralen Führen - beim Führen zur Seite - darum, für Organisationsmitglieder eine Führungskonzeption zur Verfügung zu stellen, mit denen sie auf andere einwirken, über die sie keine hierarchischen Weisungsbefugnisse haben. Dabei kommt es besonders auf die Nutzung der Einflussmechanismen Verständigung, Vertrauen und Macht an (siehe prägnant Kühl et al. 2004; siehe ausführlich Kühl 2015b).

Worin besteht das Besondere dieses Konzeptes? Und worin besteht die systematische Weiterentwicklung gegenüber anderen Konzepten? Betrachtet man die unzähligen Managementseminare über effiziente Gesprächsführung, interkulturelle Kommunikation, schlagfertige Argumentation, strategische Verhandlungsführung, erfolgreiche Mitarbeitermotivation, emotionale Führung oder diplomatisches Konfliktmanagement, dann erkennt man, dass der Schwerpunkt dieser Seminare auf der Verbesserung des Manövriergeschicks von Organisationsmitgliedern liegt. Es stehen die Fragen im Mittelpunkt, wie man Dynamiken in Gesprächen steuern kann, wie man Konflikte in Interaktionen reduziert, wie man in Gesprächen mit der eigenen Persönlichkeit überzeugt oder wie man im alltäglichen Umgang die intrinsische Motivation anderer erkennt und stimuliert (siehe Wunderer 1974 oder Klimecki 1984). Mit dem Konzept des Lateralen Führens geht es uns darum, den Fokus auf die Einflussmechanismen zu lenken, die sich hinter diesen Kniffen der Interaktion verbergen. Die Grundidee des Lateralen Führens besteht darin, Taktiken, Praktiken und Manöver der Einflussnahme vor dem Hintergrund der Prozesse in der Organisation zu betrachten.

Ziel dieses Artikels ist es, die bisherige Erfahrung mit Lateralem Führen zu resümieren und das Konzept im Hinblick auf Führung in Veränderungsprozessen voranzutreiben. Im ersten Abschnitt geht es um die drei Einflussmechanismen Verständigung, Macht und Vertrauen und ihr teilweise konfliktträchtiges Zusammenspiel. Der zweite Abschnitt widmet sich der Einbettung von Verständigungs-, Vertrauens- und Machtspielen in die formalen Strukturen von Organisationen. Laterales Führen soll Handlungsmöglichkeiten eröffnen, ohne dass die formalen Strukturen eines Unternehmens, einer Verwaltung oder eines Verbandes grundlegend geändert werden müssen. Im dritten Abschnitt werden Anwendungen des Lateralen Führens in Veränderungsprozessen - also beispielsweise bei der Entwicklung von Strategien, der Formulierung von Leitbildern, der Exploration von Märkten oder bei der Neugestaltung von Organisationsstrukturen - aufgezeigt.

\subsection{Die drei Säulen Lateraler Führung: Verständigung, Macht und Vertrauen}

Laterales Führen basiert auf drei Mechanismen der Einflussnahme: Bei Verständigung geht es darum, die Denkgebäude des Gegenübers so zu verstehen, dass neue Handlungsmöglichkeiten erschlossen werden. Vertrauen wird aufgebaut, wenn eine Seite einseitig in 
Vorleistung geht (indem sie ein Risiko eingeht) und die andere Seite dies nicht für einen kurzfristigen Vorteil ausnutzt, sondern dieses Vertrauen später erwidert. Macht spielt bei Lateralem Führen eine wichtige Rolle - nicht in der Form hierarchischer Anweisungen, sondern aufbauend auf anderen Machtquellen, wie die Kontrolle der internen, häufig informellen Kommunikation, der Einsatz von Expertenwissen oder die Nutzung von Kontakten zur Umwelt der Organisation.

\section{Zum Zusammenspiel von Macht, Vertrauen und Verständigung}

Häufig greifen Macht, Vertrauen und Verständigung so ineinander, dass sie sich gegenseitig stützen. Wenn man sich vertraut, fällt häufig auch die Verständigung leichter. Man geht zunächst einmal davon aus, dass der andere einen nicht über den Tisch ziehen will und dass es ihm darauf ankommt, unterschiedliche Einschätzungen auszutauschen. Wenn man in einer Beziehung viel Macht hat, kann man andere auch leichter ,überreden“, die eigenen Gedanken anzuhören - z. B. dadurch, dass man die anderen zu einer Sitzung ,bittet“.

Aber Verständigungs-, Macht- und Vertrauensprozesse können sich auch gegenseitig behindern. Das Aufbrechen von Denkgebäuden bringt Informationen ans Licht, die andere in Machtspielen nutzen können. Das überdeutliche Signalisieren, dass man eine für den anderen zentrale Unsicherheitszone beherrscht, kann den Aufbau von Vertrauensbeziehungen einschränken. Wenn man sich in dem Aufbau einer Vertrauensbeziehung befindet, kann es schwierig sein, eigene Interessen mit Macht durchzusetzen.

Es gibt nicht die für alle Kooperationsformen geltende richtige Mischung aus Vertrauen, Macht und Verständigung. Aber im Laufe der Analyse der Vertrauens-, Macht- und Verständigungsprozesse kann sich der Mechanismus einpendeln, über den in der konkreten Kooperationsbeziehung ein Effekt erzeugt werden kann. In der situationsabhängigen jeweils unterschiedlichen Schwerpunktsetzung auf Verständigung, Macht oder Vertrauen liegt der Clou der Lateralen Führung.

\section{Gegenseitige Ersetzbarkeit von Macht, Vertrauen und Verständigung}

Für den Prozess des Lateralen Führens ist es besonders interessant, dass sich die Verständigungs-, Macht- und Vertrauensprozesse gegenseitig wenigstens teilweise ersetzen können. Wenn sich Misstrauen in eine Kooperationsbeziehung einschleicht, dann kann es notwendig sein, neue Machtspiele zu entwickeln, mit denen man Dinge vorantreibt. Wenn man sich vertraut, braucht man nicht auch die Denkgebäude des anderen zu verstehen. Man kann in Vorleistung gehen, ohne sich im Einzelnen darüber klar zu sein, was genau den anderen treibt.

Konkret heißt dies, dass man im Lateralen Führen nach Alternativen zum jeweils dominierenden Einflussmechanismus Ausschau halten kann. Man sucht nach Prozessen, die ähnliches leisten können wie der Prozess, mit dem man im Moment nicht weiterzukommen scheint. Wenn man es mit einem eingefahrenen Machtspiel zu tun hat, kann ein Kooperationspartner durch Diskussionsführung versuchen, geschlossene Denkgebäude $\mathrm{zu}$ öffnen und so Verständigung statt Machtauseinandersetzungen zu erzielen. Dies kann entweder dazu führen, dass die Rationalität einer Entscheidung erhöht wird oder dass als Kompromiss neue Spielregeln entstehen. Wenn ein Kooperationspartner erkennt, dass er 
über Verständigungsprozesse nicht weiterkommt, weil die lokalen Rationalitäten sich zu sehr unterscheiden, kann er beispielsweise durch die Schaffung zusätzlicher Regeln neue Machtspiele eröffnen, wodurch eventuell die eine Seite sich durchsetzen kann.

\subsection{Laterales Führen - die Rückbindung an die Formalstrukturen von Organisationen}

Verständigung, Macht und Vertrauen spielen in jeder sozialen Beziehung eine Rolle. Schaut man sich Familien an, dann kann man beobachten, wie sich (blindes) Vertrauen zwischen den Ehepartnern aufbaut, wie sie bei der Erziehung um Verständigung ringen oder wie mehr oder minder subtile Machtspiele eingesetzt werden, um den Partner dazu zu bringen, das zu tun, was man von ihm erwartet. In Gruppen - Freundeskreisen, Cliquen pubertierender Jugendlicher, Straßengangs, ,,autonomen“ linken politischen Gruppen oder kleineren terroristischen Zusammenschlüssen - kann man beobachten, wie sich Vertrauen (oder Misstrauen) ausbildet, wie um Verständigung gerungen wird und wie sich Machtspiele ausbilden. Selbst bei kleinen, regelmäßigen Zusammentreffen beispielsweise beim Gemüsehändler können sich diese Mechanismen andeuten: Als treuer Kunde lässt man anschreiben, man versucht zu begreifen, weswegen ein Produkt so viel teurer geworden ist oder droht (häufig unausgesprochen) mit dem Wechsel zum neu gegründeten Grünhöker um die Ecke.

Was ist jetzt das Besondere an der Wirkungsweise von Verständigung, Macht und Vertrauen in Organisationen?

\section{Ein Gedankenexperiment - Macht, Verständigung und Vertrauen in der perfekten Organisation}

In einer perfekten, allein durch die Formalstruktur dominierten Organisation müsste man sich um Macht, Verständigung und Vertrauen keine Gedanken machen (siehe Kühl 2011). In der perfekten Organisation - so jedenfalls hier das Gedankenspiel - wäre einerseits über die Hierarchie und andererseits über die Bildung von Abteilungen klar definiert, wer in welcher Frage das Sagen hat. Bei eindeutig bestimmten Zuständigkeiten gäbe es keinen Raum für Machtspiele. In der perfekten Organisation wüsste jeder über alles Bescheid, was ihn in seiner Position betrifft. Alle würden die Denkweise und Logiken der anderen verstehen. Eine Verständigung wäre deswegen nicht mehr nötig. Es existierten keine Regelungslücken, die man durch personenbezogenes Vertrauen füllen müsste.

Der Zustand einer ,,perfekten Organisation“ mag im „,wertschöpfenden Kern“ manches Unternehmens, mancher Verwaltung oder Non-Profit-Organisation wenigstens zeitweise erreichbar sein. Die Fertigung in der Produktion, die Abarbeitung von Sozialhilfeanträgen, die Essensausgabe an Kranke oder die Versendung von Spendenaufrufen mögen manchmal so weit standardisierbar sein, dass für Machtspiele, Vertrauensprozesse und Verständigung weder die Möglichkeit noch die Notwendigkeit besteht. Möglich ist dieser durchstandardisierte „wertschöpfende Kern“ jedoch nur dadurch, dass spezialisierte Abteilungen wie 
Arbeitsvorbereitung, Lagerhaltung oder Personaler für diesen „wertschöpfenden Kern“ permanent Unsicherheiten abfedern. Die Macht-, Vertrauens- und Verständigungsprozesse verlagern sich dadurch lediglich.

\section{Verständigung, Macht, Vertrauen - Wirkung im Schatten der Formalstruktur}

Die Besonderheit von Organisationen ist, dass sie in der Lage sind, die drei Einflussmechanismen Macht, Vertrauen und Verständigung zu „formalisieren“. Es gibt Machtprozesse, die durch die Organisation abgesichert sind. Dazu gehört nicht nur die Hierarchie, sondern auch die Macht, die man über die Hierarchie verliehen bekommt - zum Beispiel der Sonderbeauftragte der Chefin, die Verhandlungsvollmachten eines Außendienstmitarbeiters oder das Recht, gegen bestimmte Entscheidungen ein Veto einzulegen. Auch Vertrauen lässt sich formalisieren. Neben dem Vertrauen in Personen gibt es auch ein Vertrauen in Organisationen (Organisationsvertrauen) - die Verlässlichkeit, dass Arbeitsverträge gelten, dass Gehälter gezahlt und notfalls eingeklagt werden können oder die Sicherheit, dass von einer bestimmten Abteilung Informationen geliefert werden, weil die Regeln das vorsehen. Ferner kann Verständigung in Organisationen in seinem sehr begrenzten Rahmen angeordnet werden, zum Beispiel durch die Einrichtung von „Regelterminen“" zur Abstimmung zwischen zwei Abteilungen.

Die für das Laterale Führen wichtigen Einflussmechanismen laufen aber eher im Schatten der Formalstruktur ab. Die Kooperationspartner mögen zwar ihre Machtquellen durch die Formalstruktur aufbauen, sichern oder ausbauen können - die Machtspiele, die ablaufen, sind dann aber eher die kleinen taktischen Spielzüge, die man einsetzt, um seine eigenen Interessen durchzusetzen. Ähnlich ist auch das Verhältnis bei Vertrauen. Organisationen mögen Wundermaschinen sein, um von der Notwendigkeit des persönlichen Vertrauens zu entlasten. Mitarbeitende folgen (wenn auch widerwillig) dem Chef, weil es die Organisation so vorsieht, und bis zu bestimmten Grenzen können sich der Chef und die Mitarbeitenden darauf verlassen. Man kann damit rechnen, dass man sein Gehalt gezahlt bekommt und ein Arbeitsvertrag Bestand hat, auch wenn die eigene Mentorin die Firma bereits verlassen hat. Aber im Schatten des Systemvertrauens spielt das Vertrauen zwischen Personen eine wichtige Rolle in Organisationen. Will der Chef von seinen Mitarbeitenden verlangen, dass sie über die reguläre Arbeitszeit hinweg länger bleiben, braucht es das Vertrauen der Mitarbeitenden, dass ihnen dieses Entgegenkommen irgendwann vergolten wird. Will man nicht nur - durch einen Arbeitsvertrag abgesichert - in der Organisation verbleiben, sondern auch Karriere machen, helfen Netzwerke, die auf dem Vertrauen zwischen Personen gegründet sind. Organisationen mögen ferner auch Verständigungsprozesse zunächst einmal überflüssig erscheinen lassen. Man kann sich erst einmal darauf verlassen, dass Dinge deswegen erledigt werden, weil die Organisation dies verlangt. Aber im Schatten laufen Prozesse ab, in denen man versucht, die Positionen des anderen zu verstehen und seine eigenen Positionen verständlich zu machen (Tab. 16.1).

Diese Macht-, Vertrauens- und Verständigungsprozesse können sich auch deswegen ausbilden, weil sie durch die Formalstruktur der Organisation nicht erzwungen, verboten oder verlangt werden können. In einer Organisation kann ,wirkliche“ Verständigung nicht 
Tab. 16.1 Macht, Vertrauen und Verständigung - Unterschiedliche Ebenen der Wirkweise

\begin{tabular}{l|l|l}
\hline Macht & $\begin{array}{l}\text { In Organisationen vorhan- } \\
\text { dene formalisierte Variante des } \\
\text { Einflussmechanismus }\end{array}$ & $\begin{array}{l}\text { Nicht formalisierte Variante des } \\
\text { Einflussmechanismus }\end{array}$ \\
\hline Vertrauen & $\begin{array}{l}\text { Macht, deren Akzeptanz zur Mit- } \\
\text { gliedschaftsbedingung gemacht wird, } \\
\text { z. B. die Hierarchie, aber auch durch } \\
\text { die Organisation - vermittelt über die } \\
\text { Hierarchie - verliehene Macht }\end{array}$ & $\begin{array}{l}\text { Macht, die auf Machtquellen wie } \\
\text { Expertenwissen, Kontakte zur Umwelt } \\
\text { der Organisation, Beherrschung von } \\
\text { informellen Kontaktwegen basiert }\end{array}$ \\
\hline $\begin{array}{l}\text { Organisationsvertrauen - das Ver- } \\
\text { trauen, dass man sich auf die Kom- } \\
\text { munikationswege und Programme der } \\
\text { Organisation verlassen kann, dass man } \\
\text { sicher sein kann, wer Mitglied der } \\
\text { Organisation ist oder auch nicht }\end{array}$ & $\begin{array}{l}\text { Organisationen. Das Vertrauen basiert } \\
\text { auf der gegenseitigen Kenntnis des }\end{array}$ \\
$\begin{array}{l}\text { Verhaltens der Personen im Rahmen } \\
\text { ihrer Mitgliedschaftsrolle (und bei- } \\
\text { spielsweise nicht auf der Kenntnis der } \\
\text { Mitgliedschaftsrolle allein) }\end{array}$ \\
\hline $\begin{array}{l}\text { Verständigung } \\
\text { Durch die offiziellen Kommunika- } \\
\text { tionswege der Organisation gedeckten } \\
\text { Verständigungsprozesse, z. B. in der } \\
\text { Form von Verständigung zwischen } \\
\text { Vorgesetzten und Untergebenen oder } \\
\text { der Regelkommunikation zwischen } \\
\text { Abteilungsleitern }\end{array}$ & $\begin{array}{l}\text { Durch die offiziellen Kommuni- } \\
\text { kationswege nicht gedeckte Ver- } \\
\text { ständigungsprozesse, z. B. die } \\
\text { Abstimmungen auf dem kurzen } \\
\text { Dienstweg, die Verständigung zwi- } \\
\text { schen subalternen Mitarbeitenden } \\
\text { verschiedener Abteilungen, bevor ein } \\
\text { Gutachten offiziell vorgelegt wird }\end{array}$ \\
\hline
\end{tabular}

erzwungen werden. Machtspiele können nicht gesetzlich verboten werden. Vertrauen zwischen Personen kann nicht hierarchisch verlangt werden.

\section{Verständigung, Macht, Vertrauen - die Begrenzung durch die Einbindung in Orga- nisationen}

Organisationen unterscheiden sich von anderen sozialen Gebilden wie Liebesbeziehungen, Freundeskreisen oder spontanen Interaktionen dadurch, dass die Macht-, Vertrauensund Verständigungsprozesse durch die Formalstruktur eingehegt werden. In Liebesbeziehungen gibt es lediglich gesellschaftliche Bremsmechanismen - besonders das Rechtswesen und die kulturellen Normen -, die Prozesse der Macht, des Vertrauens oder der Verständigung einschränken können.

Freundescliquen können weitgehend auf Vertrauen oder Verständigung basieren, und regulierte Machtverhältnisse kann es hier kaum geben. Nur im Fall von beispielsweise Gewalteskalationen greifen dann Mechanismen des Rechtssystems. Macht-, Vertrauens- und Verständigungsprozesse in Organisationen sind deswegen besonders, weil diese Prozesse durch die Formalstruktur stärker reguliert werden.

Schon ein Blick auf den Mechanismus von Vertrauen zeigt, dass die formalen Strukturen einer Organisation oftmals das Ziel haben, solche Abstimmungsprozesse zu unterbinden, die auf Personenvertrauen basieren. Die Strukturen einer Organisation, an die ihre 
Mitglieder gebunden sind, solange sie Mitglied bleiben wollen, machen es für alle erwartbar, dass Befehle befolgt, Routinen angestoßen werden oder Abstimmungen stattfinden, auch wenn man dem Gegenüber als Person nicht vertraut. Ein Blick auf Organisationen in Entwicklungsländern - man denke nur an die Wasserverwaltung in Jordanien, die Telekommunikationsunternehmen auf den Philippinen oder die Stadtentwicklungsgesellschaften im Senegal - zeigt, dass es geradezu als Pathologie einer Organisation gewertet wird, wenn das Personenvertrauen überhandnimmt und die Formalstruktur im Konfliktfall nicht die Oberhand über das Personenvertrauen gewinnen kann.

Auch Verständigung wird durch die Formalstruktur der Organisation eingehegt. Selbst wenn es den Verfechtern des Mottos „Kommunikation, Kommunikation, Kommunikation" schwer fallen mag, zu akzeptieren - Organisationen mit ihrer Formalstruktur sind erst einmal großartige Mechanismen zur Unterbindung von Verständigung. Sowohl Hierarchen als auch Vertreter in Abteilungen können sich in letzter Konsequenz auf ihre durch die Formalstruktur abgesicherte Position zurückziehen, um Aufforderungen zur Verständigung abzublocken. Der Clou der Formalstruktur von Organisationen ist erst einmal, dass sie festlegt, wem gegenüber man nicht rechenschafts- oder auskunftspflichtig ist. Die Formalstruktur kann also dafür eingesetzt werden, ausufernde Verständigungsprozesse abzukürzen.

Organisationen bilden auf den ersten Blick einen idealen Nährboden für Machtkämpfe um Ressourcen, Informationen, Zugänge oder Verantwortlichkeiten. Aber auch das Ausufern dieser Machtkämpfe wird durch die Formalstruktur reduziert. Die zentrale Funktion von Hierarchien ist, dass sie die alltägliche Neuaushandlung von Machtpositionen überflüssig machen. Im Streitfall kann der hierarchisch Höherstehende eine Auseinandersetzung mit Verweis auf die ihm durch die Formalstruktur verliehenen Rechte „formal“ entscheiden. Es ist also die Formalstruktur der Organisation, die eine ungehemmte Ausdehnung von Machtspielen unterbindet.

\section{Verständigung, Macht und Vertrauen - die Veränderung der Spiele über Organisa- tionsstrukturen}

Eine soziologisch aufgeklärte Führungslehre würde den Blick zunächst einmal auf die Veränderung der Formalstruktur richten. Die Stärke einer hierarchischen Führung ist die Veränderung von Formalstrukturen - also die Zusammenlegung oder Trennung von Abteilungen, die Festlegung von neuen Zielen, die Veränderung von Arbeitsabläufen oder die Entlassung beziehungsweise Einstellung von Mitarbeitenden.

Interessant ist, dass die Zielrichtung des Lateralen Führens häufig nur unterhalb dieser Ebene der Veränderung von Formalstrukturen einsetzt. Es werden kleine Mechanismen des Gebens und Nehmens etabliert, im Bereich der Informalität angesiedelte Macht-Tauschbörsen eingerichtet oder Verständigungsprozesse jenseits der formal vorgeschriebenen Abstimmungsmechanismen etabliert. Durch den Prozess des Lateralen Führens verändern sich häufig nur die alltäglich praktizierten Routinen, die Ansichten des Personals oder die ungeschriebenen Ziele einzelner Akteure. 
Diese kleinen Veränderungen im Rahmen des Lateralen Führens werden dann (wenn überhaupt) häufig erst später durch die Organisation formalisiert. Die Hierarchie segnet eine bestimmte alltägliche Praxis ab, ein implizites, aber für die Organisation funktionales Ziel wird zur offiziellen Zielvorgabe, oder ein in einem Bereich langsam aufgebautes Wissen wird darüber kodifiziert, dass dieser Bereich offiziell zum Kompetenzzentrum für bestimmte Fragen erklärt wird.

Aber über diese Frage hinaus kann das Konzept des Lateralen Führens auch dazu eingesetzt werden, um die Struktur der Organisation neu zu gestalten.

\subsection{Macht, Vertrauen und Verständigung in Veränderungsprozessen}

Ein besonderer Anwendungsfall des Konzeptes des Lateralen Führens sind Prozesse des geplanten Wandels von Organisationen (also das, was man früher Organisationsentwicklung nannte und was heute häufig auch im deutschsprachigen Kontext etwas hochtrabend als Change Management bezeichnet wird). In vielen Organisationen lassen sich Reorganisationen nicht allein über die Hierarchie durchsetzen. Häufig besitzt die Spitze der Organisation gar nicht die Informationen, um eine Anpassung von Organisationsstrukturen vorzunehmen, und ist deswegen zur Einbindung subalterner Mitarbeitender gezwungen. Häufig können aber auch die Organisationsmitglieder im operativen Bereich die von Expertenberatern ersonnenen und von der Hierarchie abgesegneten neuen Organisationsstrukturen im organisatorischen Alltag zerreiben und die Konzepte so zu reinen Papiertigern verkommen lassen. Auch deswegen werden Mitarbeitende breit an der Planung der neuen Veränderungsprozesse beteiligt.

In diesen Fällen greift das Konzept des Lateralen Führens, weil es zwar die zentrale Funktion von Hierarchien in Organisationen anerkennt, auf hierarchische Steuerung aber weitgehend verzichtet. Somit können, wenn Eingriffe von Hierarchien eher selten möglich sind, auch Reorganisationen vorangebracht werden. Gleichzeitig ist aber dieser Anwendungsfall besonders problematisch, weil hier nicht nur die Routinen des alltäglichen Arbeitens beeinflusst werden, sondern vor allem deswegen, weil die Rahmenbedingungen, unter denen kooperiert wird, verändert werden.

Der Wandel von Abteilungszuschnitten, hierarchischen Zuordnungen oder Standardprozeduren führt auch zu einer Veränderung der lokalen Rationalitäten. Damit verändern sich - wenn auch langsam - sowohl die Denkgebäude als auch die Interessen der Akteure. Dadurch verändern sich Verständigungsprozesse. Ferner werden durch den Wandel der Formalstruktur die Machttrümpfe neu verteilt. Abteilungen gewinnen oder verlieren $\mathrm{Zu}-$ gänge zu Wissensressourcen, zu wichtigen externen Spielern oder zu Kommunikationskanälen innerhalb der Organisation. Die Ausgangsbasis für zukünftige Machtspiele wird gelegt. Weiterhin gibt es noch keine Erfahrungen mit den geplanten neuen Zuständen der Organisation. Vertrauen muss sich unter diesen Bedingungen teilweise erst wieder neu bilden. Für die betroffenen Mitglieder einer Organisation steht bei diesen Reorganisationen also besonders viel auf dem Spiel. 
Tab. 16.2 Klassische versus Laterale Führung von Veränderungsprozessen

\begin{tabular}{l|l|l}
\hline & $\begin{array}{l}\text { Klassische Vorgehensweise im } \\
\text { Management von Veränderung }\end{array}$ & $\begin{array}{l}\text { Vorgehensweise im Rahmen } \\
\text { des Konzepts des Lateralen } \\
\text { Führens beim Management von } \\
\text { Veränderung }\end{array}$ \\
\hline $\begin{array}{l}\text { Phase: Analyse der } \\
\text { Ist-Situation }\end{array}$ & $\begin{array}{l}\text { Identifizierung von „Widerständlern“ } \\
\text { und Entwicklung von Strategien im } \\
\text { Umgang mit ihnen }\end{array}$ & $\begin{array}{l}\text { Verzicht auf das Konzept des } \\
\text { „Widerstandes“ gegen Wandel } \\
\text { - Routinelogik ist, genauso wie } \\
\text { Innovationslogik, lediglich eine } \\
\text { lokale Rationalität innerhalb der } \\
\text { Organisation }\end{array}$ \\
\hline $\begin{array}{l}\text { Phase: Gestaltung von } \\
\text { Interaktionen }\end{array}$ & $\begin{array}{l}\text { Diskursive Aufdeckung der bestehen- } \\
\text { den Macht-, Verständigungs- und } \\
\text { Vertrauensverhältnisse }\end{array}$ & $\begin{array}{l}\text { Akzeptanz der Latenz von } \\
\text { Macht-, Verständigungs- und } \\
\text { Vertrauensverhältnissen }\end{array}$ \\
\hline $\begin{array}{l}\text { Phase: Implementie- } \\
\text { rung von Lösungen }\end{array}$ & $\begin{array}{l}\text { Offenhaltung lediglich in der Phase } \\
\text { der Problemlösung und der Lösungs- } \\
\text { erarbeitung. Danach Schließung der } \\
\text { Kontingenz und Umsetzung der ver- } \\
\text { abschiedeten Lösungen }\end{array}$ & $\begin{array}{l}\text { Offenhalten von Kontingenz- } \\
\text { Erprobungen, bevor zu Ende } \\
\text { gedacht wurde }\end{array}$ \\
\hline
\end{tabular}

Es wäre naiv, die Planung neuer Strukturen von Organisationen einzig und allein mit den Kategorien von Verständigung, Macht und Vertrauen erfassen zu wollen. Bei der Planung neuer Organisationsstrukturen - also bei der Entscheidung über die Prämissen zukünftiger Entscheidungen - spielen ganz andere Fragen eine Rolle. Es geht auch (und man könnte behaupten vorrangig) darum, wie die Kommunikationswege, die Programme und das Personal der Organisation zukünftig ineinander greifen sollen, welche Aufgaben zukünftig ausschließlich innerhalb der Organisation und welche in Kooperation mit anderen Organisationen erledigt werden sollen, und welche neuen Kooperationsformen sich zwischen den Beteiligten ausbilden sollen. Aber mit den Kategorien Macht, Vertrauen und Verständigung kann man den Blick auf einige wichtige Aspekte von Veränderungsprozessen öffnen (dies ist - zugegebenermaßen etwas karikaturell - in Tab. 16.2 zusammengefasst und wird nachfolgend erläutert).

\section{Die Ausgangssituation: Innovations- und Routinelogiken durch Transmission ver- knüpfen}

Die sich aus den ,alten“ Organisationsstrukturen ergebenden lokalen Rationalitäten spielen eine wichtige Rolle bei der Analyse von Macht-, Vertrauens- und Verständigungsprozessen im Zuge der Veränderung von Organisationsstrukturen. Darauf bauen die unterschiedlichen Denkgebäude auf, die die Verständigung untereinander erschweren oder erleichtern, ebenso die Vertrauens- (oder Misstrauens-) beziehungen und die Machtarenen und Machtspiele.

Eine auf den Status quo gerichtete Analyse von Macht-, Vertrauens- und Verständigungsprozessen wird noch durch unterschiedliche Logiken in den Veränderungsprozessen 
ergänzt, erweitert und teilweise sogar überlagert. Zum Beispiel arbeiten die Anführenden von Veränderungsprozessen häufig mit einem relativ einfachen Differenzierungsschema: Auf der einen Seite ständen die „Innovatoren“, die ein Unternehmen, eine Verwaltung oder ein Krankenhaus zu neuen Ufern führen wollen, Personen also, die einem Wandel aufgeschlossen gegenüberstehen. Auf der anderen Seite befänden sich die ,Widerständler“, die für den „Status quo“ stehen und die Organisation um den notwendigen Wandel bringen. Diesen Personen wird dann häufig eine anerzogene, wenn nicht sogar angeborene Neigung zur Stabilität unterstellt. Bei dieser Herangehensweise handelt es sich aber lediglich um eine Verabsolutierung der spezifischen lokalen Rationalität, die dann durch Vorträge und Veröffentlichungen von „Change Agents“, ,Veränderungsmanagern“ oder von „Gurus des Wandels“ ideologisch abgesichert wird.

Für Laterales Führen in Veränderungsprozessen ist es notwendig, mit der gleichen Sorgfalt, mit der beispielsweise die jeweiligen lokalen Rationalitäten von unterschiedlichen Funktionsbereichen rekonstruiert werden, auch die lokalen Rationalitäten von verschiedenen Interessengruppen in Veränderungsprozessen zu analysieren. Erst auf der Basis der Rekonstruktion dieser lokalen Rationalitäten können dann die Vertrauensroutinen, die Verständigungsprozesse und die Machtspiele verstanden werden. Es fällt dann schnell auf, dass der Drang zum geplanten Wandel keine quasi naturgegebene Notwendigkeit der Organisation ist, sondern dass sich die positive Haltung zum geplanten Wandel vielmehr aus der Position innerhalb der Organisation ergibt.

Die Logik der Innovation wird häufig von bestimmten Bereichen innerhalb von Organisationen vorangetrieben - vom Topmanagement, von den Stabsstellen für Strategie und Organisation und natürlich von den für die Durchführung von Veränderungen eingekauften und an deren erfolgreicher Umsetzung gemessenen Beratern. Diese Personen sind dann innerhalb der Organisation „Symbole des Wandels“ - ein dauernder Verweis darauf, dass man es auch anders machen könnte. In dieser Logik der Innovation dominieren Vorstellungen von der Veränderung bestehender Routinen, von großen umfassenden Konzepten und von deren konfliktfreier, standardisierter Implementation. Diese Logik der Innovation wird noch verschärft durch die für die „Innovatoren“ charakteristischen Vorstellungen ,professionellen Arbeitens“, durch monetäre Gratifikationen für „erfolgreich abgeschlossene Wandlungsprozesse“ und durch Belohnungen in Form von Karrieresprüngen.

Dem gegenüber steht - darauf hat besonders Günther Ortmann (1995) aufmerksam gemacht - die Logik von Routinen, die häufig von den Organisationsmitgliedern im operativen Bereich verfolgt wird. Ihnen liegt besonders die Stabilisierung der alltäglichen Arbeitsabläufe beispielsweise in der Materialwirtschaft, in der Produktionssteuerung, in der Fertigung und Montage oder im Vertrieb am Herzen. Ihre Vorstellungen von Professionalität, ihre Gratifikationen und ihre Karrierechancen sind - aller Managementrhetorik des Wandels zum Trotz - zu erheblichen Teilen an die erfolgreiche Aufrechterhaltung dieser Routinen gebunden. Insofern vertreten sie eher eine Logik der inkrementalen Verbesserungen, der Sicherheit der Prozesse und der Beachtung von Bereichsspezifika. 
Es gibt keinen Anlass, die Logik der Innovatoren höher einzuschätzen als die Logik der Routine-Vertreter. Die Diskriminierung der Routine-Vertreter als „Widerständler“ oder „Low-Performer" mag für die Stabilisierung innerhalb einer Gruppe von Innovatoren vielleicht eine wichtige Rolle spielen, verbaut aber letztlich Chancen, die lokalen Rationalitäten in einem Veränderungsprozess als Ansatzpunkte für die Veränderung von Macht-, Vertrauens- und Verständigungsprozessen heranzuziehen.

Auf dem Terrain der Veränderungsprojekte treffen häufig die Vertreter einer Rationalität der Innovation und die Vertreter einer Rationalität der Routine aufeinander. In den Veränderungsprojekten findet dann ein „Transmissionsspiel“ der Vertreter mit ihren unterschiedlichen Logiken statt. Erst dieses häufig konfliktreiche „Transmissionsspiel“ ermöglicht es, dass die Innovationen nicht ein Hirngespinst von Topmanagern, Stabsstellen oder Beratern bleiben, sondern - wenn auch in veränderter Form - Niederschlag in den alltäglichen Praktiken der Organisation finden.

\section{Die Besprechungsphase: Die Latenz lateraler Führungsbeziehungen akzeptieren}

In der Change-Management-Literatur wird häufig die ,,schonungslose Analyse“ und ,diskursive Aufdeckung“ der bestehenden Situation in der Organisation gefordert. Erst auf der Basis einer genauen Aufdeckung der Macht-, Vertrauens- und Verständigungsprozesse in der Organisation sei es möglich, Veränderungsimpulse gezielt zu setzen. Die ,wirklichen Abläufe" in einer Organisation müssten in Workshops aufgearbeitet und kritisch diskutiert werden.

Bei dieser wegen seinem aufklärerischen Impetus sicherlich sympathischen Position wird jedoch übersehen, dass Macht-, Vertrauens- und Verständigungsprozesse sich im „Latenzbereich“ (ein Begriff aus der soziologischen Systemtheorie) von Organisationen befinden. Das heißt, selbst bei bewusster Wahrnehmung von Teilansichten dieser Prozesse durch einzelne Personen, kann die Gesamtbeschaffenheit der Macht-, Vertrauens- und Verständigungsprozesse nicht eindeutig identifiziert und kommuniziert werden.

Ein wichtiger Unterschied zwischen der Formalstruktur einer Organisation und den bei Lateraler Führung wirksamen Macht-, Vertrauens- und Verständigungsprozessen besteht in der Möglichkeit bzw. in der Unmöglichkeit, in Diskussionsprozessen eindeutig Bezug darauf zu nehmen. Aspekte der Formalstruktur - die offiziellen Kommunikationswege, die verabschiedeten Programme und die verkündeten Personalentscheidungen - sind in Organisationen in der Regel problemlos identifizierbar und damit eindeutig ansprechbar. Man kann auf seinen hierarchischen Status verweisen, um eine Entscheidung durchzusetzen oder sich auf die Zielsetzungen oder Wenn-Dann-Regeln der Organisation beziehen. Viele Macht-, Vertrauens- und Verständigungsprozesse laufen jedoch eher in der Informalität der Organisation ab, können sich nicht auf formale Abstützungen der Organisation berufen und sind deswegen nicht eindeutig identifizierbar oder offen ansprechbar.

Die Kommunikationslatenz hat aus systemtheoretischer Sicht die Funktion des ,Strukturschutzes“ für die informellen Macht-, Vertrauens- und Verständigungsprozesse. Der Aufbau von Vertrauen zwischen Personen kann durch offenes Ansprechen (,,ich vertraue dir") oder gar offenes Einklagen (,vertrau mir doch") beeinträchtigt werden. Machtspiele 
verändern sich, wenn diese offen thematisiert werden, und Machtquellen, die nicht durch die Formalstruktur abgesichert sind, können an Kraft verlieren, wenn sie für alle sichtbar aufgedeckt werden. Auch die informellen Verständigungsprozesse in Organisationen können häufig nur deswegen ablaufen, weil - jedenfalls offiziell - niemand anderes davon weiß.

Wie soll mit dieser Latenz vieler Vertrauens-, Macht- und Verständigungsprozesse in Veränderungsprozessen umgegangen werden?

Die existierenden Macht-, Vertrauens- und Verständigungsprozesse zwischen bestimmten Kooperationspartnern lassen sich in der Regel im Gespräch mit lediglich einem dieser Kooperationspartner gut erschließen, denn Kommunikationsschwellen, die bei Anwesenheit des häufig mit anderen Sichtweisen und Interessen ausgestatteten Gegenübers bestehen können, entfallen. Solche Schwellen sind zum Beispiel daran erkennbar, dass das Thematisieren eines besonderen Vertrauensverhältnisses von einem oder sogar beiden Kooperationspartnern mit einem genervten Gesichtsausdruck quittiert wird oder die Existenz einer nicht durch die Formalstruktur abgedeckten Machtquelle geleugnet wird. Die Gestalter von Veränderungsprozessen werden also sehr gut überlegen, ob sie existierende Vertrauens-, Macht- oder Verständigungsprozesse außerhalb von Vier-Augengesprächen aufdecken oder nicht.

Als Berater erhält man deswegen häufig nur dann einen guten Einblick in die existierenden Macht-, Vertrauens- und Verständigungsprozesse, wenn man zwischen Gesprächen mit Personen mit weitgehend homogenen Auffassungen oder Rollen (z. B. Vertreter einer Abteilung) oder Gesprächen mit Personen mit sehr heterogenen Auffassungen (z. B. Vertreter unterschiedlicher Abteilungen oder Organisationen) hin- und herwechseln kann. Beim letztgenannten Gesprächstypus ist das Ansprechen der latenten Macht-, Vertrauensund Verständigungsprozesse eine riskante Intervention, auf die häufig mit Negierung, Abweisung oder Aggression reagiert wird.

\section{Zur Anlage von Veränderungsprozessen: Der Nutzen der Kontingenz}

Im klassischen zweckrationalen Organisationsmodell, das lange Zeit auch die Organisationsentwicklung dominierte, ist die Anlage von Veränderungsprozessen relativ übersichtlich. Aus einer klaren Definition des Zwecks einer Organisation lasse sich - nach einer genauen Bestimmung der Umweltbedingungen - das Ziel eines Veränderungsprozesses - die „,beste Lösung“ für die Organisation - definieren. Unter Beteiligung möglichst vieler Betroffener sei dieses Ziel dann in die durch verschiedene Subeinheiten handhabbaren Unterziele zu zerlegen. Veränderungsprojekte müssten dabei, so die Annahme, in abgrenzbare Projektphasen wie Problemdiagnose, Konzeption, Spezifikation und Implementierung unterteilt werden. Unter einer Phase wird dabei in der Regel ein in sich abgeschlossener Arbeitsabschnitt verstanden, der mit einem überprüfbaren Meilenstein endet.

Diese idealtypisch propagierte Vorgehensweise wird dann aber häufig schon in der organisatorischen Praxis nicht durchgehalten. Schon die Einigung darauf, was eigentlich die „beste Lösung“ ist, gestaltet sich schwierig. Die „beste Lösung“ fällt in der Regel unterschiedlich aus - je nachdem, aus welcher Perspektive innerhalb der Organisation man auf 
das vermeintliche Problem schaut. Niemand - auch die Hierarchie nicht - kann ,neutral“ beurteilen, welche Lösung besser ist als die andere. Selbst wenn offiziell eine gemeinsame Lösung verkündet wird, wird diese häufig noch in der Implementierungsphase zerrieben, weil sie sich nicht in die existierenden Machtverhältnisse einzupassen scheint.

Angesichts solcher Gegebenheiten empfiehlt sich eine Vorgehensweise, die auf eine vergleichsweise lange Offenhaltung der „Kontingenz“ basiert. Unter Kontingenz versteht man dabei, dass ein Ereignis nicht notwendig ist, sondern auch anders möglich wäre. Aus der Analyse eines Problems A ergibt sich nicht zwangsweise die Lösung X, sondern möglicherweise auch die Lösung Y oder die Lösung Z.

Häufig wird der Fehler gemacht, dass frühe Festlegungen getroffen werden, die einer späteren Kooperation im Wege stehen. Durch frühe Festlegungen entstehen zwar Konzepte, zu denen alle Beteiligten Lippenbekenntnisse ablegen, die sich dann aber allzu schnell als Planungsruinen entpuppen.

Die Kontingenz in Veränderungsprozessen kann man darüber sichtbar halten, dass Lösungen lediglich als Erprobungen eingeführt werden. Bei der Erprobung können mehrere unvollständige, auch widersprüchliche Konzepte gleichzeitig angestoßen werden. Schließlich gehört es zu den Stärken der Organisation, dass sie auch widersprüchliche Herangehensweisen verkraften kann.

Der Vorteil der Erprobungen besteht darin, dass sich in einem durch das „Signum der Vorläufigkeit“" geschützten (Zeit-)Raum neuartige Verständigungs-, Vertrauens- und Machtprozesse entwickeln. Das häufig neu zusammengewürfelte Personal im reorganisierten Feld kann im Rahmen der veränderten Kommunikationswege und Programme Erfahrungen miteinander machen und gegenseitiges Vertrauen (oder auch Misstrauen) entwickeln. Häufig bilden sich durch die wenn auch nur probeweise übernommenen neuen Positionen andere Rationalitäten aus, über die neuartige Verständigungsmöglichkeiten entstehen. Weil sich in dem als Probe ausgeflaggten Kooperationsfeld auch die Machtquellen neu verteilen, können sich auch Machtprozesse zwischen den Kooperationspartnern neu gestalten.

Durch das Ausprobieren verschiedener Lösungen kann die eine oder andere ,,abstürzen“, wenn sie sich als nicht tragbar erweist. Eine Lösung kann aber auch durch die Erprobung an Qualität gewinnen, wenn die Umsetzung Erfolg versprechend ist. Manchmal entstehen durch die Erprobung auch neue Stoßrichtungen, die bislang nicht beachtet wurden. Geeignete Lösungen kristallisieren sich heraus.

\section{Literatur}

Klimecki, R. (1984). Laterale Kooperation - Grundlagen eines Analysemodells in horizontaler Arbeitsbeziehungen in funktionalen Systemen. Bern: Paul Haupt.

Kühl, S. (2011). Organisationen. Eine sehr kurze Einführung. Wiesbaden: Springer.

Kühl, S. (2015a). Sisyphos im Management. Die verzweifelte Suche nach der optimalen Organisationsstruktur (2. Aufl.). Frankfurt a. M.: Campus. 
Kühl, S. (2015b). Laterales Führen. Macht, Vertrauen und Verständigung in Organisationen. Wiesbaden: Springer.

Kühl, S., Schnelle, T., \& Schnelle, W. (2004). Führen ohne Führung. HarvardBusinessManager, 1, 71-79. (kann unter quickborn@metaplan.com angefordert werden).

Ortmann, G. (1995). Die Form der Produktion. Organisation und Rekursivität. Opladen: WDV.

Wunderer, R. (1974). Lateraler Kooperationsstil. Personal, 8, 166-170.

Prof. Dr. Stefan Kühl (geboren 1966) ist Professor für Organisationssoziologie an der Universität Bielefeld und arbeitet seit 1992 als Organisationsberater bei Metaplan. Er berät Unternehmen, Verwaltungen, Ministerien und Universitäten besonders in Fragen von Strategie- und Organisationsentwicklung. Er ist Autor des Lehrbuchs „Organisationen. Eine sehr kurze Einführung“./ Dr. Thomas Schnelle (geboren 1954) ist Soziologe und Erkenntnistheoretiker. In seiner Dissertation setzte er sich mit dem Werk Ludwik Flecks auseinander. Seit 1983 ist er Metaplaner, heute als Geschäftsführender Partner. Er berät Managementgremien zu Vertriebs-, Strategie- und Organisationsfragen. Schwerpunktmäßig arbeitet er für die Pharmaindustrie, Beratungs- und Technologieunternehmen. 